\title{
Diversidad y reconocimiento de la producción académica en los sistemas de evaluación de la investigación en Colombia
}

\author{
Gabriel Vélez-Cuartas*, Henry Gómez-Flórez*, Ana Úsuga-Ciro*, Manuel Vélez-Trujillo* \\ * Departamento de Sociología. Facultad de Ciencias Sociales y Humanas. Grupo de Investigación Redes y Actores Sociales. Universidad de \\ Antioquia. Medellín. Colombia. Correo-e: gabrielvelezcuartas@gmail.com
}

Recibido: 04-11-2013; 2a version: 18-12-2013; Aceptado: 08-01-2014.

Cómo citar este artículo/Citation: Vélez-Cuartas, G.; Gómez-Flórez, H.; Úsuga-Ciro, A.; Vélez-Trujillo, M. (2014). Diversidad y reconocimiento de la producción académica en los sistemas de evaluación de la investigación en Colombia. Revista Española de Documentación Científica, 37(3):e056. doi: http://dx.doi.org/10.3989/redc.2014.3.1133

Resumen: Este artículo hace una revisión de los productos resultados de investigación reconocidos por las seis universidades mejor posicionadas en Colombia en los rankings internacionales y los documentos de Sistema Nacional de Ciencia, Tecnología e Innovación. Se utiliza análisis de redes exploratorio y estadística descriptiva para analizar la producción documental de las instituciones con respecto a sus metas de producción investigativa y evaluación de su desempeño. Se demuestra que la discusión internacional acerca de lo que debe o no medirse para dar cuenta del impacto de la investigación académica y científica aún hace invisibles muchas formas de producción que revelan construcción de nuevo conocimiento.

Palabras clave: Comunicación de la investigación; análisis de redes; documentación institucional; comunicación académica; producción científica; sistemas de medición; cienciometría.

\section{Diversity and recognition of academic production in Colombia's research evaluation systems}

Abstract: This article reviews both those products resulting from research processes in the 6 best ranked universities in Colombia according to international rankings, and documents from the National System of Science, Technology and Innovation. Exploratory network analyses and descriptive statistics are used to analyse the institutions' documents with respect to their research production goals and performance evaluations. The international discussion about what should and what should not be measured in order to determine the impact of scholarly and scientific research is shown not to take into account many forms of new knowledge production, resulting in such products remaining invisible

Keywords: Communication of research; network analysis; institutional documentation; academic communication; scientific production; measurement systems; scientometrics.

Copyright: ( 2014 CSIC. Este es un artículo de acceso abierto distribuido bajo los términos de la licencia Creative Commons Attribution-Non Commercial (by-nc) Spain 3.0. 


\section{INTRODUCCIÓN}

En la página web de la Asociación Alemana de Sociología (2013) se presenta un listado del 70\% de las universidades alemanas (entre ellas las más importantes) que tienen departamento de Sociología y declaran que no quieren hacer parte del ranking universitario del CHE (la organización que construye el U-Multirank). Esta situación se puede considerar como uno de los tantos síntomas para describir los descontentos con el desempeño de los rankings universitarios en el mundo (Colectivo para la Ciencias, 2013; Dorrë, 2013). Es importante tener en cuenta que la sociología nació en ese país y sigue produciendo algunos de los documentos más importantes en el campo.

Es cierto que cada vez que aparece un ranking también aparece como mínimo un crítico de éste. Por ejemplo alguno que no quedó entre los primeros lugares. Sin embargo, cuando la medición ya no sólo opera como mecanismo de inclusión o exclusión de investigadores o instituciones, sino también de campos científicos enteros, de países con trayectoria investigativa, de publicaciones de calidad certificadas por asociaciones nacionales o regionales, de investigadores fundamentales para diferentes campos, hay algo más que pareciera no estar funcionando bien (Nederhof, 2006; Rubio Liniers, 1999; Hazelkorn, 2010; Macías Chapula, 2001).

Algunos problemas evidenciados ocurren por ejemplo en las ciencias sociales y humanidades que concentran el grueso de su producción más destacada en libros (Archambault y Larivière, 2010). Los libros no tienen bases de datos consistentes y por lo tanto quedan sin registro gran cantidad de obras relevantes, como ya lo habían evidenciado Glänzel y Schoepflin (1999) algunos años atrás y aún lo denuncian Giménez y Torres-Salinas (2011). Si bien ISI y Scopus están haciendo un esfuerzo por vincular otras formas de producción en productos como el Book Citation Index, por ejemplo, las editoriales incluídas por estas bases de datos continúan siendo las de las grandes universidades de habla inglesa, o importantes trasnacionales como Elsevier, Wiley, etc., dejando también por fuera parte importante de la producción no registrada en estas grandes editoriales (Torres Salinas, 2013; Vélez Cuartas y otros, 2013b).

Otro problema se evidencia en la cobertura. Rodríguez Yunta (2010) evidenció de manera precisa los subregistros de publicaciones Iberoamericanas en WOS y Scopus. De manera general, Latindex considera en su catálogo cerca de 20.000 revistas iberoamericanas en contraste con las cerca de 500 presentes en las bases de datos ISI y Scopus en el 2010. Pero estas bases no presentan sólo problemas de cobertura, sino también en la delimitación de sus alcances. Por ejemplo, Michels y Schmoch (2012) encuentran que es imposible establecer una correlación exacta entre el crecimiento de la ciencia y el crecimiento de las bases de datos WOS.
De otro lado, Sanz-Casado y otros (2013) advierten sobre la baja capacidad de observación de las dinámicas nacionales que ofrecen los rankings internacionales. La existencia de múltiples observatorios nacionales como el Atlas de la Ciencia Mexicana (http://atlasdelacienciamexicana.org/), el Observatorio IUNE en España (http://www.iune. es/), el Observatorio Colombiano de Ciencia y Tecnología (http://www.ocyt.org.co) y otras múltiples experiencias nacionales dan cuenta de mediciones más finas de la dinámica institucional. En la misma vía de la construcción de indicadores más finos, aparecen algunos índices más recientes como el ranking de editoriales de libros científicos en España y otros con una trayectoria de un poco más de una década como Publindex en Colombia para la vigilancia de la calidad de revistas nacionales. Estas bases de datos e índices evidencian la pertinencia de la diversificación en las formas de medición con respecto a las bases de datos bibliográficas e índices internacionales.

Es importante anotar que esta diversificación permite evidenciar carencias de los sistemas internacionales. En el caso específico de Publindex, Romero y otros, (2013), encuentran inconsistencias al hacer un contraste entre las revistas colombianas reconocidas por este índice y las presentes en WOS y Scopus. Los investigadores descubren que algunas revistas de calidad reconocidas por Publindex, con las mismas condiciones y características de madurez, no se encuentran en bases de datos como WOS y Scopus y otras sí. Igualmente Estrada y Forero (2010), descubren revistas de calidad que no cumplen con algunos criterios de las bases de datos internacionales para ser indizadas pero tienen una alta difusión internacional a través de los sistemas Open Journal. Google Scholar es planteado hoy como una alternativa para solucionar estos problemas, sin embargo, en una revisión profunda se encuentra que los datos no son transparentes, ni la empresa da cuenta exacta de los procedimientos que permiten el rastreo de la información (Cabezas y Torres, 2012; Delgado y Cabezas Clavijo, 2013).

En resumen se puede decir que la medición de la ciencia y el conocimiento emergente de la investigación académica enfrenta problemas complejos como son la carencia de bases de datos globales fiables para todos los campos de conocimiento; el no reconocimiento de otras formas de comunicación del conocimiento académico investigativo; y el establecimiento de puentes metodológicos y teóricos entre las situaciones nacionales y regionales de la ciencia (micro) y las dinámicas globales (macro). Este artículo se interesa especialmente por el segundo problema, el del reconocimiento de productos en los sistemas de observación y medición de la investigación académica y científica. Si bien las críticas se han centrado en la no observación de productos publicados como los libros u otras publicaciones seriadas, aquí se sostiene que el problema es aún más profundo, y que existen muchos más tipos de productos emergentes de la investi- 
gación académica que se pueden considerar como comunicación de nuevo conocimiento, pero aún no son visibles al debate internacional al respecto de lo que puede ser considerado como tal.

Para resolverlo, se han consultado los sistemas de valoración de la producción de conocimiento de las 6 universidades más visibles de Colombia en los rankings internacionales, así como los documentos gubernamentales que rigen el Sistema Nacional de Ciencia, Tecnología e Innovación. Los resultados presentados en este documento dan cuenta de: (1) los tipos de productos reconocidos como productos de conocimiento e investigación; (2) las diferencias en la valoración de estos productos por parte de las instituciones observadas; (3) las bases de datos y fuentes utilizados para hacer inventarios de la producción; (4) y los sistemas de evaluación de la producción investigativa y de conocimiento utilizados.

El objetivo general de este trabajo es identificar todas las formas de comunicación del conocimiento reconocidas por los sistemas de evaluación y seguimiento de la producción académica e investigativa en Colombia. El cumplimiento de este objetivo permite la construcción de una línea para la construcción en etapas posteriores de esta investigación de herramientas para la medición de la producción de conocimiento. Se recogen los sistemas más importantes de reconocimiento de los productos de investigación: el de las Universidades y el del Sistema Nacional de Ciencia, Tecnología e Innovación liderado por Colciencias.

\section{ESTRATEGIA METODOLÓGICA Y MATERIALES}

Los resultados fueron obtenidos a través de una estrategia de investigación documental que combina técnicas exploratorias de búsqueda de información y análisis cuantitativos. Se consideran los documentos que regulan la asignación de estímulos por productividad académica en las Universidades y en el Sistema Nacional de Ciencia, Tecnología e Innovación liderado por Colciencias. En el caso de Colciencias se han tomado tanto el modelo anterior $y$ el vigente que en el momento de escribir este artículo estaban en período de transición. Es decir, las directrices del modelo anterior vigente desde el 2008 hasta noviembre de 2013 (reemplazado por el actual) operan como mecanismo de clasificación para la producción investigativa en Colombia, hasta los resultados de la aplicación del modelo de medición que se tendrán disponibles luego de ser escrito este artículo. El Decreto 1279 estipula la asignación de estímulos a la producción académica en las Universidades Públicas del país. Este Decreto fue publicado en el año 2002 y continúa vigente. Se consideran también las normas particulares de las Universidades privadas vigentes al momento de realizar este estudio.

A continuación la descripción de las fases de recolección de información y análisis.
Delimitación y recolección de información: Se seleccionaron 41 documentos institucionales correspondientes a la planeación y evaluación de la investigación en las 6 universidades más grandes en producción y mejor posicionadas del país según los rankings de Shangai, Times Higher Education, QS world, Scimago y Webometrics. A continuación se indican los nombres de las instituciones, su fecha de creación, las páginas web institucionales y su carácter público o privado: Universidad Nacional de Colombia (1867, http://www.unal.edu.co/, pública), Universidad de Antioquia (1803, http:// www.udea.edu.co/, pública), Universidad del Valle (1945, http://www.univalle.edu.co/, pública), Universidad de los Andes (1948, http://www.uniandes. edu.co/, privada), Universidad del Rosario (1653, http://www.urosario.edu.co/, privada) y Pontificia Universidad Javeriana de Bogotá (1623, http:// puj-portal.javeriana.edu.co/, privada). Igualmente se consideraron las leyes, decretos, acuerdos y resoluciones del orden nacional relacionadas con el Sistema de Ciencia, Tecnología e Innovación (CTI), principalmente producidos por Colciencias (http://www.colciencias.gov.co/, el Departamento Nacional de Planeación (http://www.dnp.gov.co/ y el Observatorio Colombiano de Ciencia y Tecnología (http://www.ocyt.org.co/). Por parte de las Universidades se consideraron los documentos institucionales en materia de ciencia, investigación y validación de producción de conocimiento (el listado de los documentos aparece adjunto en la bibliografía). Toda la documentación fue recabada de las páginas Web Institucionales y verificada vía telefónica con las unidades respectivas en caso de duda sobre la vigencia y actualidad.

Se construyó una base con cuatro tipo de datos: (1) los registros de referencias a las diferentes formas de comunicación de los resultados de investigación y conocimiento reconocidas; (2) las escalas de valoración de los productos considerados por cada institución; (3) los sistemas de evaluación de la investigación y la producción de conocimiento mencionados por los documentos de planeación y reglamentación de las instituciones; (4) y las bases de datos utilizadas para hacer inventarios de sus productos.

Todos los productos mencionados en (1) o (2) son considerados por las universidades como producción académica vinculada en muchos casos al ejercicio de la investigación o al desarrollo de conocimiento e igualmente se conservaron los nombres de las tipologías de productos y clasificación de estos en la forma en que figuraban en los documentos de las instituciones mencionadas. El Decreto 1279 y las normatividades privadas reglamentan el estímulo a sus docentes e investigadores por productividad académica. Esa productividad se define en términos de tipos de productos sin calificar su carácter epistemológico o metodológico y se consideran por igual trabajos de carácter científico, técnico, artístico, humanístico o pedagógico. Podría decirse que estas instituciones estimulan la 
producción de conocimiento y no sólo la producción investigativa en sentido estricto.

Desde otro punto de vista, Colciencias (2013b), en su último modelo de medición 2013, caracteriza de manera más explícita la producción como investigativa y propone 4 categorías: -1- Nuevo conocimiento: referido a formas de producción: artículos en revistas indexadas en ciertas bases de datos, libros y capítulos de libro publicados por editoriales de prestigio, patentes y variedades vegetales y animales.; -2- Desarrollo tecnológico e innovación: productos tecnológicos certificados, empresariales, consultoría y leyes o normas; -3- Apropiación social y circulación de conocimiento: participación ciudadana, estrategias pedagógicas, participación en eventos y redes de conocimiento, comunicación social del conocimiento y reconocimientos; -4- Formación de recursos humanos: tesis, proyectos de grado, $\mathrm{I}+\mathrm{D}+\mathrm{i}$ con empresas, extensión y responsabilidad social, apoyo a programas de formación. Todas son consideradas actividades de investigación y son calificadas como formas de especialización de la producción investigativa. Sin embargo el modelo no aclara la participación de otras formas investigativas provenientes del terreno de las artes, la arquitectura, el diseño o algunas ramas de las ciencias de la comunicación, las cuales también tienen grupos de investigación inscritos en el sistema Scienti de registro oficial de toda la producción investigativa colombiana.

En este sentido, hay gran cantidad de actividades producidas en los grupos de investigación de estas áreas que queda en una especie de limbo en donde no está claro si lo que produce un grupo de investigación en teatro al hacer un trabajo etnográfico para realizar una adaptación de una obra teatral es un producto de investigación o de desempeño profesional. Según la definición de Colciencias, al ser actividad de grupo debería quedar catalogada como actividad de investigación dentro de uno de los 4 subgrupos. Es verdad que pueden producir un artículo, pero en realidad el producto de conocimiento es la adaptación y aunque está catalogada dentro de la clasificación de Colciencias como otro tipo de producción, no recibe valoración. Sin embargo, las normatividades de las universidades la reconocen. Este tipo de investigación, llamada investigación-creación (Moreno Vargas, 2012) es válida como producto investigativo tanto como el descubrimiento de una nueva forma de conformación molecular desde el punto de vista de las definiciones institucionales y desde el punto de vista de la tipología documental que se están considerando en este trabajo.

Se conservará de manera general el término producción de conocimiento o comunicación de la producción de conocimiento investigativo como equivalente. Es cierto que no todos los productos realizados por los profesores universitarios pueden ser considerados como productos de investigación, pero todos los tipos son susceptibles de ser toma- dos en cuenta por resultados de investigación de grupos de las diferentes áreas. Así lo corroboran los documentos de trabajo del área de ciencias sociales, artes y humanidades de la Universidad de Antioquia (2012) y algunos documentos utilizados en esta investigación de la Universidad del Valle y la Universidad de los Andes.

Análisis: se hizo un análisis de redes exploratorio para procesar la base de datos construida. Se construyeron tres redes y se calcularon valores básicos de centralidad. A mayor centralidad de grado de los datos institucionales (tipo de producto, base de datos consultada o ranking consultado), mayor su relevancia relativa para el sistema teniendo en cuenta que la población escogida es pequeña (no irrelevante en términos cualitativos). Cuanto mayor es el grado de las instituciones mayor el tipo de productos académicos e investigativos, considerados o reconocidos en sus documentos prospectivos y evaluativos (Freeman, 1978/1979). Para el procesamiento de la información se utilizó el software Pajek 3.13 (De Nooy y otros, 2011) y VosViewer 1.5.4 (www.vosviewer.com).

Igualmente, se hizo una tabla comparativa de los valores asignados a los tipos documentales en los sistemas de valoración institucional. Se construyó una escala de valores normalizados y se contrastaron: los considerados por las instituciones, por el Decreto 1279, por el modelo actual de medición de grupos del 2013 y por el anterior del 2008.

\section{RESULTADOS}

\subsection{Tipos de productos de conocimiento re- conocidos}

Se consideran productos reconocidos, aquellos que las normatividades internas de las Universidades y los documentos del Sistema Nacional de Ciencia, Tecnología e Innovación consideran como productos académicos y resultados de investigación respectivamente y a los cuales se les asigna algún tipo de puntaje para estimular o evaluar a los grupos de investigación e investigadores. En la totalidad de documentos institucionales revisados aparecen 135 tipos de productos que pueden ser reconocidos en, al menos, una de las instituciones. De éstos, 75 pueden ser considerados como productos propios de la investigación en las áreas de artes y de las ciencias de la comunicación o ciencias de la información (ver figura 1). También aparecen más de una docena de productos técnicos y tecnológicos, así como varios asociados a la forma impresa del libro (capítulos, traducciones, libro resultado de investigación, etc.), entre otros. En una primera mirada se podría decir que el sistema es amplio y diverso en lo respectivo a los tipos de producción investigativa y académica reconocidos. Se necesita, sin embargo, hacer una observación más detallada sobre el alcance de este reconocimiento, y para esto hay que tener en cuenta que 
en Colombia hay dos grandes formas de reconocer la producción a través de puntajes:

- El Modelo de Medición de Grupos de Investigación Científica, Desarrollo Tecnológico e Innovación de Colciencias 2008 y uno nuevo que está comenzando a regir desde finales del 2013 y reemplaza al anterior.

- Los criterios de medición interna de cada Universidad: para las públicas el decreto 1279 de 2002 y otras disposiciones internas que especifiquen párrafos del decreto; para las privadas documentos autónomos.

Los únicos tipos de productos reconocidos por todas las Universidades y Colciencias son los artículos en revista indexada, los libros de investigación y de texto. Igualmente la patente excepto por la Universidad del Rosario. También se destacan: creación artística original, creación artística complementaria o de adaptación, interpretación artística, artículos de revisión, y reportes de caso, cartas al editor y editorial en revistas indexadas. Se puede decir entonces que al reunir los productos que tienen algún puntaje en los documentos revisados se identifican 135 diferentes, pero al observar cuáles son reconocidos universalmente o por la mayoría de instituciones y sistemas analizados la lista se puede reducir a los mencionados en este párrafo.

De acuerdo a los mecanismos de reconocimiento interno de las instituciones, la mayor variedad en términos de producción figura en el área de artes (aproximadamente 70 tipos diferentes); sin embargo, ninguno de estos productos se encuentra estipulado dentro del modelo de medición de grupos y, por lo tanto, queda fuera de los parámetros de reconocimiento para la investigación que lleva a cabo Colciencias para todas las universidades. De todos modos se debe reconocer que ya se inició un proceso para integrar productos relacionados con arte, diseño y arquitectura (Colciencias, 2013a) (Ver figura 1).

La U. del Valle y la U. de los Andes reconocen más tipos de producción en artes que las otras universidades o modelos de medición. En el caso de la U. de los Andes esto hace parte de un proceso de creación de parámetros para reconocer la producción en esta área equivalente al que existe para productos como artículos, libros, capítulos, normas y patentes como lo han expresado ya en documentos académicos publicados (Villaveces Cardoso y otros, 2007; Villaveces Cardoso y otros, 2008; Villaveces Cardoso y otros, 2010; Villaveces Cardoso y otros, 2012). La U. del Valle publicó un documento (Resolución N 112 de 2006) en el que deja explícito los cerca de cincuenta tipos de productos que consideran pertenecientes al campo de las artes, contribuyendo a una mayor especificación de los contenidos en el Decreto 1279. A diferencia de la U. del Valle, las universidades de Antioquia y Nacional, que también son públicas y se rigen por el Decreto, dejan sin especificar qué producción ar- tística validan dentro de ellas, así que no se cuenta con información explícita y concreta de la producción que están validando en el campo de las artes (excepto por lo que figura en el Decreto).

Al contrastar los dos grandes modelos de validación puede advertirse una desarticulación entre el modelo actual de Colciencias para medir los grupos y las formas de reconocimiento al interior de las universidades. Sin embargo, el modelo de evaluación de Colciencias en discusión propone 28 tipos de actividades nuevas que no se encuentran en los documentos de validación de la producción al interior de las Universidades. La diferencia comparativa entre el modelo vigente y el Decreto 1279 en específico es mayor, ya que sólo hay 5 productos en común entre estos dos documentos: Patente, Artículo en revista indexada, Libro de investigación, Libro de texto y Software, que en realidad son los tipos de productos más comúnmente indizados y ordenados en rankings globales o nacionales.

Los dos tipos de sistemas nacionales de reconocimiento de la producción están muy separados entre sí; y es esperable que haya diferencias entre ellos ya que no responden a propósitos exactamente iguales (Colciencias posiciona grupos de investigación mientras que las universidades reconocen la producción de sus docentes); sin embargo, el nivel tan alto de esta disparidad implica que la mayoría de los productos que un investigador elabore le va a dar puntos en un sistema pero no en el otro. Lo que lleva a preguntar si esto se debe a que la investigación (Modelo de Medición de Grupos) y la producción de conocimiento de los docentes (decreto 1279) se están concibiendo como cosas muy diferentes entre sí.

Doce de 135 tipos de productos son considerados en común por todas las universidades y modelos de medición como productos de investigación. Los otros 114 dejan una pregunta importante a los sistemas de evaluación de la producción de conocimiento. La situación se complica si se observa que cada uno de estos productos no responde a características regionales de la producción de conocimiento, sino a las características de disciplinas y campos especializados concretos que albergan procedimientos propios como la investigación creación, la investigación para la realización de traducciones modernas y antiguas, la investigación filosófica, la investigación teórica, la investigación matemática de modelos teóricos, etc.

\subsection{Diferencias en la valoración de productos}

Los 135 productos referenciados en los documentos institucionales se pueden identificar diferentes puntuaciones para 60 . Éstas son valoradas con un puntaje determinado en la reglamentación que reconoce la producción académica e investigativa de los investigadores en las universidades y en Colciencias. Esta valoración se presenta en la tabla I en contraste. 
Figura 1. Producción académica reconocida por instituciones colombianas

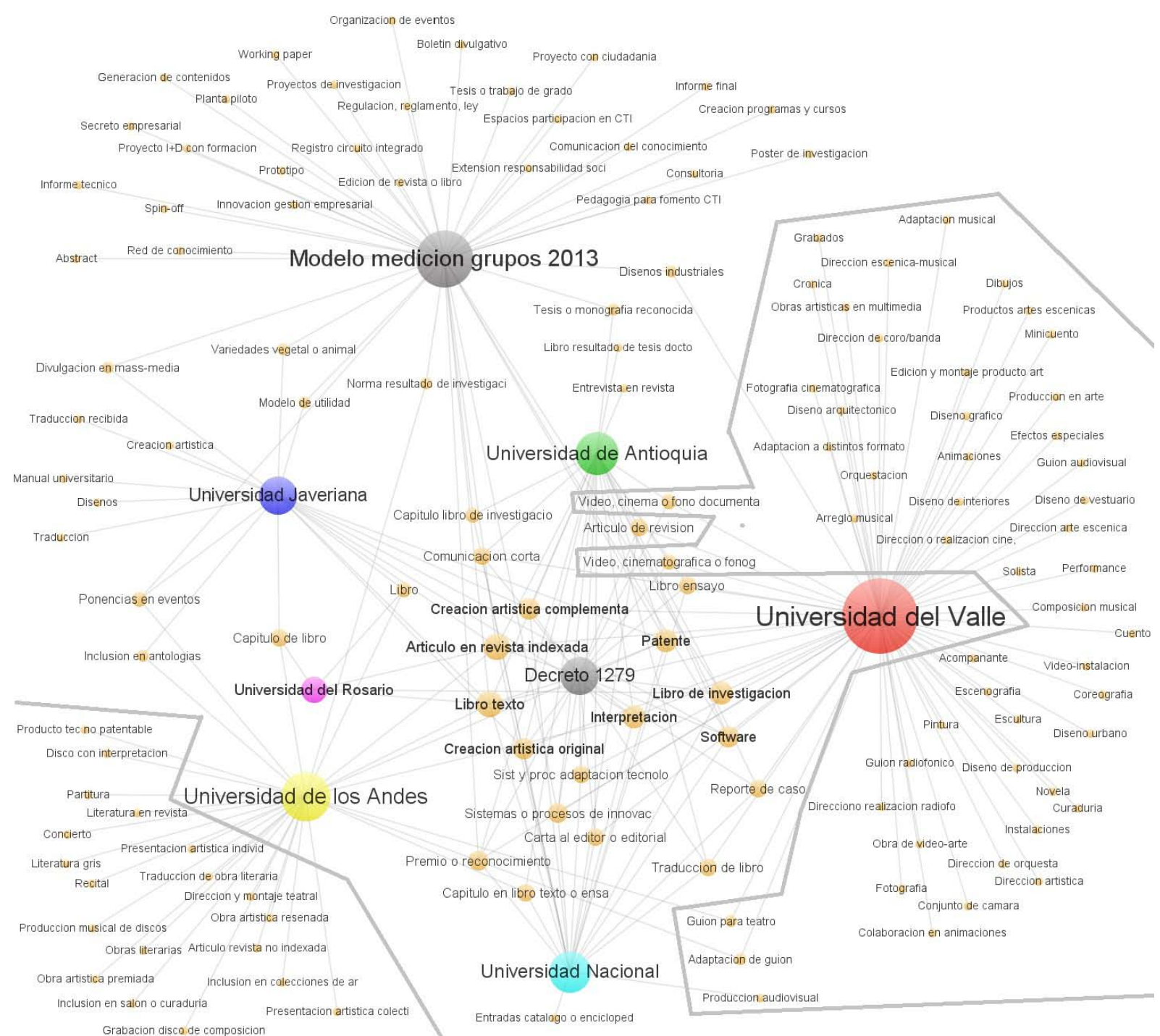

Tomada y corregida de Vélez Cuartas y otros (2013a).

Nodos: Universidades, Modelo Colciencias, Decreto 1279 y Tipos de productos. Relaciones: Validación o consideración del producto dentro del sistema de evaluación. Tamaño de los nodos: Número de relaciones (Para las Universidades el tamaño significa el número de tipos de productos validados, para los productos la cantidad de instituciones por las que son considerados válidos.). Los nodos agrupados por la línea gris corresponden a productos frecuentes en las áreas de ciencias de la comunicación y artes.

En los 60 se agrupan en 3 categorías 75 de los productos que figuran en la figura 1 correspondientes a (1) creación original, (2) interpretación y (3) apoyo a obra artística. (1) Creación original: diseño arquitectónico, diseño urbano, diseño de interiores, escultura, escultura, pintura, grabados, dibujos, instalaciones, performance, obra de video-arte, video instalación, obra en multimedia, guión audiovisual, guión radiofónico, dirección y realización de cine, TV y video, dirección y realización radiofónica, fo- tografía cinematográfica, efectos especiales, diseño de vestuario, escenografía, animaciones, producción en arte, edición y montaje de producto artístico, diseño de producción, dirección artística, composición musical, guión para teatro, dirección arte escénica, coreografía, diseño gráfico, novela, cuento, crónica, minicuento, texto dramático. (2) Interpretación: curaduría, adaptación de guión, adaptación a distintos formatos, colaboración en animaciones, dirección de orquesta, dirección coro o banda, dirección escénica- 
musical, solista, conjunto de cámara, acompañante. (3) Complementaria y apoyo: arreglo musical, orquestación, adaptación musical, transcripción (Resolución No 112 de 2006 de la Universidad del Valle). Igualmente: Obras literarias, dirección teatral, montaje teatral, presentación artística individual, presentación artística colectiva, inclusión en salón o curaduría, inclusión en colección de arte, disco con interpretación, producción musical de discos, partitura, grabación de disco con composiciones propias, obra artística premiada, traducción de obra literaria, obra artística reseñada, interpretación, concierto, recital (Villaveces Cardoso y otros, 2010). Se optó por no incluirlos uno por uno en la tabla, sino agruparlos por las categorías señaladas. El resto de productos sí se desglosan con su respectivo puntaje debido a la heterogeneidad de su puntaje.

Se observa que todas las instituciones universitarias, sean públicas o privadas, valoran, en conjunto, sólo 3 productos: libro de investigación, artículo en revista A según el índice de Publindex y Libro de texto. Es importante aclarar que la categoría A se ha generalizado para las revistas presentes en índices ISI y Scopus u otros índices de relevancia internacional. Las universidades públicas (U. de Antioquia, U. Nacional y U. del Valle) otorgan la misma importancia al libro de investigación, puntaje que varía en la valoración que hacen de él las universidades privadas (Universidad Javeriana y Universidad del Rosario), siendo en la Universidad del Rosario, por ejemplo, el producto más valorado. Para el caso del Modelo de Medición de Grupos del año 2008, se le otorga un importante valor en comparación con el artículo en revista $A$ y con el libro de texto.

Las patentes son el producto de más alta valoración, excepto en la Universidad del Rosario que no las contempla (sus carreras pertenecen al campo de las ciencias sociales y humanidades). Los artículos en revistas indexadas son valorados por todas las Universidades investigadas, pero no son los de mayor valor en el sistema. Los libros, comprendiendo por ellos, el libro de investigación, el libro de texto, la traducción de libro, el capítulo de libro y el libro de ensayo, tienen diferencias valorativas entre universidades (Ver Tabla I).

En lo referido a la producción de Artes en las Universidades Estatales, se evidencia que sus formas de comunicación son altamente valoradas. Esto se observa en el reconocimiento de la Creación Artística Original con idéntico valor al del Libro de Investigación, inclusive por encima del artículo en revista A. La Obra Artística Complementaria, la menos valiosa de las tres producciones de Artes, es más valorada por las Universidades Estatales que el Artículo en revista B. Sin descuidar que el Decreto 1279 permite a las Universidades Estatales clasificar la producción de las Artes en cualquiera de las tres categorías, se evidencia una importante valoración de la producción Artística, valoración que permite a los investigadores de esta área com- parar su producción investigativa con áreas ajenas a las suyas, teniendo igual valor en ciertos productos específicos. En cuanto a las universidades privadas, la Universidad Javeriana valora también la Creación y la Interpretación Artísticas de igual manera que el Libro de Investigación y que el Artículo en Revista A respectivamente. La Obra Artística Complementaria equivale en ella a la Traducción de un libro. Empero, la Universidad del Rosario (que no tiene programa de artes) y el modelo de medición 2008 no validan ni valoran la producción Artística, excluyendo de sus objetivos esta forma de producción de conocimiento.

La Universidad de los Andes llevó acabo el inventario, la clasificación y la valoración de toda su producción en libros, para lo cual, además de revisar en su propia biblioteca y centros de investigación, terminó de completar la información con otras cinco fuentes más. A falta de un referente que reuniera toda su información en este tipo de producción hicieron uno propio, y el documento de conceptualización del proceso intitulado "La publicación de libros de investigación en la Universidad de los Andes: una propuesta de criterios editoriales y científicos". Este documento inclusive sirvió como referente conceptual a Colciencias para la construcción del sistema que está permitiendo registrar los libros a nivel nacional. Son procesos de recuperación de la producción propia, pero construidos bajo estándares internacionales de Harvard University Press, Cambridge University Press y Oxford University Press, que visibilizan resultados que de otra forma se hubieran perdido.

De manera general, las universidades públicas que siguen el decreto 1279 dan un alto valor a la diversidad de productos académicos. Las universidades privadas limitan más su espectro. El modelo anterior de Colciencias también de carácter público como el decreto, es mucho más restringido que éste. El modelo actual de Colciencias es mucho más generoso, considera un abanico inmenso de productos con alta valoración. Se podría apelar a un argumento vocacional para explicar esta situación por la especialización disciplinar de las instituciones, pero en una mirada con lupa, es posible encontrar el conflicto latente en decisiones administrativas inconsistentes que promueven en los planes de trabajo la visibilidad en bases de datos como Scopus o WOS, pero que en su normatividad y legislación premian una gama de productos mucho más amplia.

Esta situación tiene dos caras: una es un sistema dinámico en búsqueda de adaptar mecanismos de valoración de las formas de comunicación dentro de un contexto institucional; tal vez no siempre con los mejores resultados, pero definitivamente dinámico. Otra, y más importante que la anterior, los mecanismos de valoración ponen en evidencia las deficiencias de los sistemas que valoran la comunicación del conocimiento investigativo. Hay muy pocos productos valorados por los rankings internacionales y no represen- 
tan la totalidad de las formas de comunicación del conocimiento, ni pueden considerarse como representativos de ésta. Si bien los estándares internacionales de investigación reconocen muy pocos productos de acuerdo a las bases de datos disponibles en el mercado para inventariar la producción, las Universidades y el sistema nacional, comienzan a observar una gama amplia de posibilidades de comunicar conocimiento académico y resultado de investigación. De ninguna forma puede decirse que son nuevas formas de comunicar el conocimiento, pero sí representan una novedad para los sistemas globales de evaluación y valoración mucho más recientes, que la misma historia del conocimiento producido de manera académica (Stichbe, 2003).

Tabla I. Comparación entre las formas de valoración de la producción de nuevo conocimiento

\begin{tabular}{|c|c|c|c|c|c|c|c|}
\hline PRODUCTOS & U. ANTIOQUIA & U. NACIONAL & U. VALLE & U. JAVERIANA & U ROSARIO & MODELO 2008 & MODELO 2013 \\
\hline Patente & 25 & \begin{tabular}{|r|}
25 \\
\end{tabular} & 25 & \begin{tabular}{r|}
200 \\
\end{tabular} & & 4 & 10 \\
\hline Libro de investigación & 20 & 20 & 20. & 100 & 3815000 & 3 & 10 \\
\hline Artículo en revista científica & 15 & 15 & 15 & 100 & 3052000 & 1 & 10 \\
\hline Libro de texto & +2 & 15. & 15. & 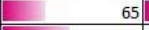 & 2670500 & 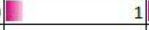 & 10 \\
\hline Creación artistica original & 20. & 20. & 20 & \begin{tabular}{|l|ll} 
& \\
\end{tabular} & & & \\
\hline Software & 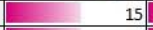 & 言 & 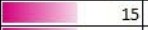 & & & & 10 \\
\hline Sistemas o procesos de innovación & 15 & 15 & 15 & & & 2 & \\
\hline Tesis o monografía con alto reconocimiento & \begin{tabular}{|l} 
\\
\end{tabular} & & & & & $\square$ & 10 \\
\hline Interpretación artística & D & 14 & 14. & 100 & & & \\
\hline Traducción de libro & 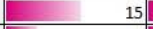 & 15 & 15 & 40 & & & \\
\hline Capitulo de libro & $\square$ & 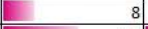 & & Proporcional & 381500 & 0.6 & 10 \\
\hline Libro de ensayo & L & 15 & 15 & & & & \\
\hline Premio o reconocimiento & 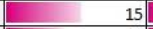 & $\square$ & 15 & 25 & & & 10 \\
\hline Obra artística complementaria o de apoyo & 12. & 12. & 12 & 40 & & & \\
\hline Videos, cinematográficas, fonográficas & 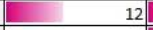 & 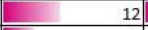 & $\square$ & & & & \\
\hline Ponencia & & - & & 10 & & & 10 \\
\hline Creación programas y cursos de formación & & & & & & 1 & 10 \\
\hline Consultorías & & & & & & 1 & 10 \\
\hline Regulación, norma, reglamento, ley & & & & & & & 10 \\
\hline Spin-off & & & & & & $P$ & 10 \\
\hline Comunicación corta & 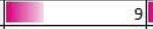 & \begin{tabular}{|lll} 
\\
\end{tabular} & $\square$ & & & & \\
\hline Poster de investigación & & & & & & & 10 \\
\hline Organización de evento & & & & & & & 10 \\
\hline Acompañamiento y asesoríasprograma Ondas & & & & & & & 10 \\
\hline Extensión de responsabilidad social & & & & & & & 10 \\
\hline Innovación en la gestión empresarial & & & & & & & 10 \\
\hline Pedagogía para fomento $\mathrm{CTI}$ & & & & & & & 10 \\
\hline Proyecto ID+I con formación & & & & & & & 10 \\
\hline Estrategia de comunicación del conocimiento & & & & & & & 10 \\
\hline Participación ciudadana en proyectos $\mathrm{CTI}$ & & & & & & & 10 \\
\hline Redes de conocimiento & & & & & & & 10 \\
\hline Variedad vegetal o animal & & & & & & & 10 \\
\hline Proyecto de investigación y desarrollo & & & & & & & 10 \\
\hline Working papers & & & & & & & 10 \\
\hline Modelo de utilidad & & & & 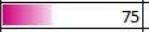 & & & 6 \\
\hline Libro resultado de tesis doctoral & 20 & & & & & & \\
\hline Diseño industrial & & & & & & & 8 \\
\hline Reporte caso & 4.5 & 4.5 & 4.5 & & & & \\
\hline Artículo de revisión & 4.5 & 4.5 & 4.5 & & & & \\
\hline Carta al editor o editorial & 4.5 & 4.5 & 4.5 & & & & \\
\hline Edición revista o libro investigación & & & & & & & 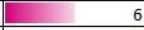 \\
\hline Norma resultado de investigación & & & & & & D & \\
\hline Divulgación en mass media & & & & 5 & & & \\
\hline Informe técnico & & & & & & & 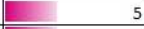 \\
\hline Espacios de participación ciudadana en CTI & & & & & & & $\square$ \\
\hline Generación de contenidos & & & & & & & 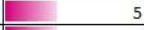 \\
\hline Secreto empresarial & & & & & & & 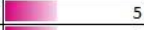 \\
\hline Tesis o trabajo de grado & & & & & & & 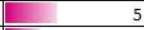 \\
\hline Esquema de circuito integrado & & & & & & & 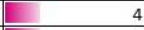 \\
\hline Planta piloto & & & & & & & 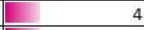 \\
\hline Prototipo industrial & & & & & & & $\square$ \\
\hline Abstracts & & 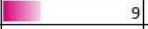 & & & & & \\
\hline Boletín divulgativo de investigación & & & & & & & 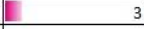 \\
\hline Servicio técnico & & & & & & 1 & \\
\hline Literatura circulación restringida & & & & & & 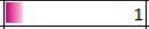 & \\
\hline Manual universitario & & & & E & & & \\
\hline Producto registrado & & & & E & & & \\
\hline Informe final & & & & & & & 2 \\
\hline Inclusión en antologías y colecciones & & & & 25 & & & \\
\hline Entrada catálogo científico o enciclopedia & & & & & & & \\
\hline
\end{tabular}

Tomada y corregida de Vélez Cuartas y otros, (2013a)

Nota a la Tabla 1: Rangos de valoración: Universidades Públicas 1-25; Modelo de medición 2013 Colciencias 1-10; Modelo de Medición 2008 Colciencias 0.1-4; U. Javeriana 5-200; U. Rosario 381500-3815000. Se realizó una normalización de la valoración para hacer contrastables los valores asignados aasignados por cada universidad. Las barras de cada celda representan la valoración normalizada respecto al valor máximo otorgado por cada institución al producto, lo que permite comparar las asignaciones valorativas de cada institución y el peso con respecto a las valoraciones generales. La Universidad de los Andes no hace mención a una escala cuantitativa de puntuación de los productos en los documentos consultados. 


\subsection{Bases y fuentes para inventariar la producción}

¿Cuál de esta producción se encuentra registrada o almacenada para ser susceptible de ser medida? Los documentos pueden dar la posibilidad de asignar puntaje a cada uno de los tipos de productos investigativos, pero si no hay bases o repositorios donde se pueda ingresar dicha producción, no hay forma de establecer todas las capacidades y potencialidades que se están generando en la investigación y en el trabajo académico. Este apartado responde a la pregunta ¿si se fuera a hacer un inventario de la producción de cada institución a qué bases de datos o informes recurriría?

La primera respuesta es simple y sin sorpresas para las instituciones colombianas: ISI Thompson, Scopus, ScienTI y Publindex. Por instituciones aquí se han considerado no sólo las universidades y los modelos de medición de Colciencias sino también las leyes, documentos de planeación nacionales del gobierno central (CONPES por ejemplo) y del Departamento Nacional de Estadística (DANE). Estos documentos serían los que regirían en gran parte la normatividad del SNC\&T+I. La visualización de la figura 2 permite ver el contraste y la concentración que hay en este aspecto.

Cabe señalar que las bases ISI y Scopus cubren una cantidad importante de producción, si se entiende que las formas más estandarizadas de comunicación del conocimiento son el artículo y los otros tipos de productos que habitualmente se publican en las revistas científicas y libros. Scopus además de los artículos contempla más de 360 colecciones de libros según su sitio web en agosto de 2013. ISI por su parte tiene una colección de 40000 libros aproximadamente en el Book Citation Index consultado en la misma fecha. Estas cantidades aún no reflejan parte importante de la producción del conocimiento por este medio, pero es información que está indizada y disponible para su consulta para quien pague sus servicios (GiménezToledo y Torres-Salinas, 2011). Pese a la cobertura, el límite en la variedad de formas de comunicación del conocimiento deja por fuera a más de 100 tipos de productos de los que se hallan en la figura 1. Es importante considerar que ni Publindex, ni ScienTI son índices bibliográficos de citación. El primero clasifica las revistas, el segundo permite consultar y valorar la información de la producción de los grupos registrados en Colombia. Este último sistema tiene el mismo problema que todos los sistemas regionales o nacionales de inventarios de la producción investigativa: son imprecisos por la forma en que se recoge la información.

Es importante aclarar que los tres primeros tipos de referentes son útiles para evaluar la productividad de los investigadores y sus planes de trabajo, así mismo como para construir rankings de productividad y hacer uso de ellos para la generación de reconocimientos. El ScienTI es especialmente utilizado por las universidades para verificar la ac- tividad de un grupo y su existencia institucional habilitando la posibilidad de participar en concursos por recursos o convocatorias.

Los problemas de incompletud de los registros en las bases de datos más consultadas han llevado a la U. de los Andes y la U. del Valle a diversificar sus fuentes de consulta. La primera acude especialmente a otros registros nacionales adelantados por la Bibliotecas Nacional y Luis Ángel Arango, busca su producción en tiendas globales como Amazon para verificar su impacto en la distribución y observa otras fuentes muy importantes como Ulrich Periodicals o Google Books. La U. Nacional considera bases de datos nacionales e internacionales de patentes, registros del Instituto Colombiano Agrario en el campo de las ciencias agrarias y bases de datos de la producción de investigadores como Academic Search.

\subsection{Fuentes de medición de impacto de la producción investigativa}

La Universidad Nacional de Colombia es, en el país, la que más se ha encargado de realizar una caracterización y comprensión del sistema mundial de rankings haciendo un inventario exhaustivo de las características, de su procedencia, del año de aparición y de variables e indicadores que miden, además de saber de dónde toman los datos para construir sus indicadores (Sánchez Torres y otros, 2011). En la figura 3 se visualiza esto en la referencia de la Universidad Nacional a 22 rankings mundiales. Sólo 8 de ellos son tenidos en cuenta por otras universidades.

La Universidad de los Andes, observa menos rankings para la construcción de indicadores que la Universidad Nacional, pero todos ellos se ubican como centrales; al ver la figura 3 se puede apreciar que esta universidad se encuentra muy alineada con los que están marcando la pauta. Y algo que se puede ver en los textos mismos es que estas referencias no son hechas sólo con fines informativos, sino con el propósito de aclarar qué es lo que debe hacer la Universidad para llegar a figurar en esos espacios. En el tipo de documentación que se revisó, las otras universidades no realizan ejercicios amplios de exploración de las posibilidades de observación de múltiples rankings.

Si bien los rankings analizados en este apartado dan cuenta de diferentes aspectos y variables correspondientes al desarrollo institucional y educativo de las universidades, nos ha interesado especialmente, indagar por el tipo de referencias utilizadas para observar la productividad. Así, el análisis de referencias a rankings contó con una revisión de los tipos de productos considerados. Se ha encontrado que los tipos de productos que son reconocidos en los rankings analizados por las universidades estudiadas se encuentran sólo revistas, patentes y libros, el resto de producción no tiene impacto en los rankings.

Los rankings toman como principales fuentes de información las bases de datos Web of Science, 
Figura 2. Bases y fuentes para inventariar la producción

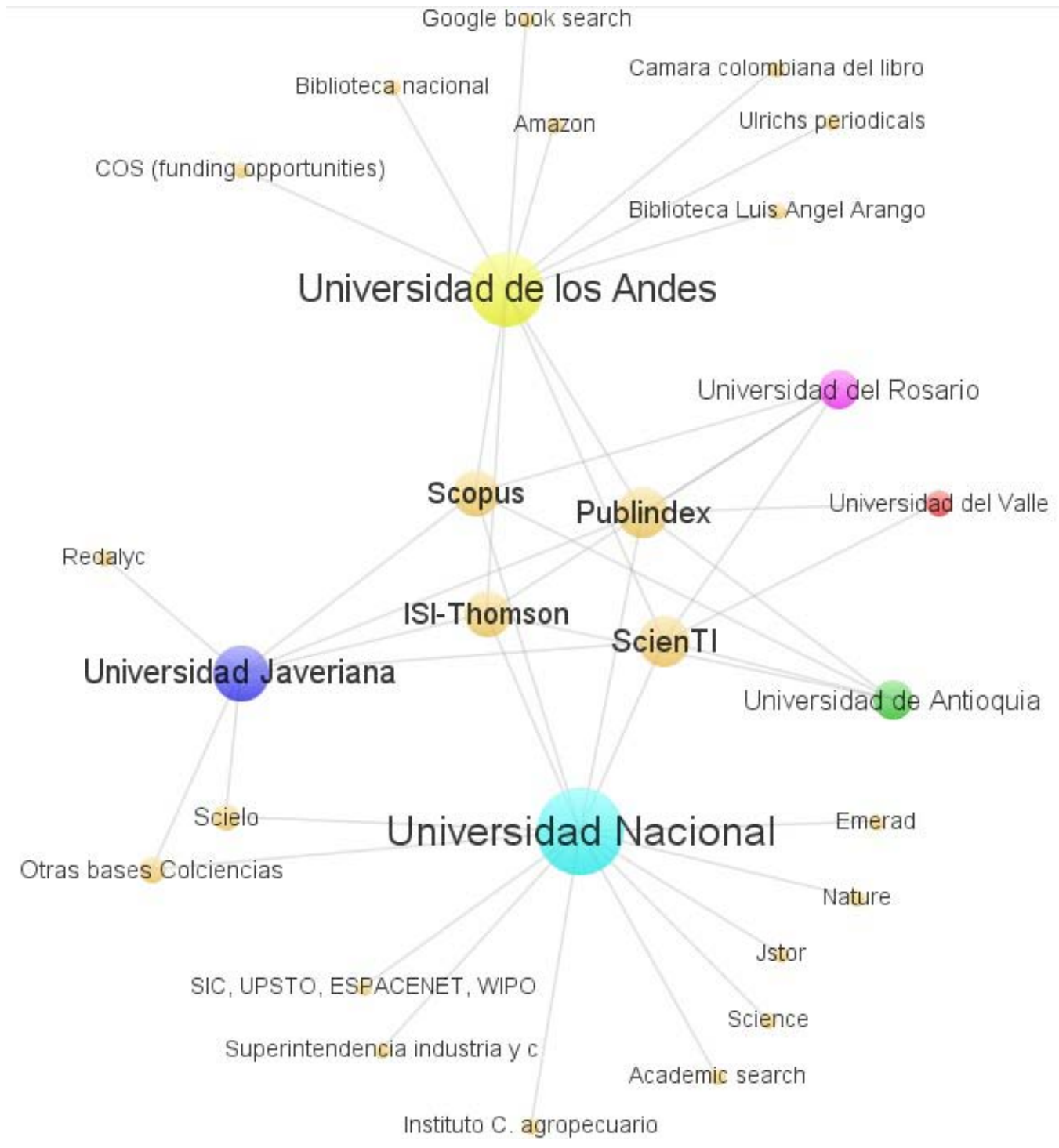

Tomada y corregida de Vélez Cuartas y otros, (2013a).

Nodos de colores: Universidades; Nodos blancos: Documentos del SNC\&T+I; Nodos negros: Fuentes de información citadas. Relaciones: citación de las fuentes de información en documentos institucionales.

Scopus y el Book Citation Index. Con este último sistema, ISI Thompson procura recuperar los registros relevantes o de mayor impacto en las ciencias sociales, en tanto el $60 \%$ de los registros pertenece a esta área, incluyendo humanidades y artes tal y como se anuncia en su sitio web. Si bien Scopus se especializa en indexación de revistas, también contiene libros en su base de datos, por lo que un sistema de rankings basados en ISI y Scopus estaría midiendo la productividad a partir de revistas, pero también en parte de la producción en libros. Otro tipo de productos considerados son las bases de datos de patentes internacionales, aunque en una menor medida que las revistas.

La posición de las universidades en los rankings es algo que quizá le interese mucho a sus administradores, sobre todo por el efecto en la imagen que obtienen sus instituciones y los logros en la gestión. De esta manera no es difícil pensar que muchas universidades concentren sus esfuerzos en mejorar el tipo de productividad, que está siendo tomada como variable por estos rankings, y que se terminen alineando a sus formas y resultados, inclusive con sus limitaciones. 
Figura 3. Fuentes de medición de impacto de las 6 Universidades mejor posicionadas

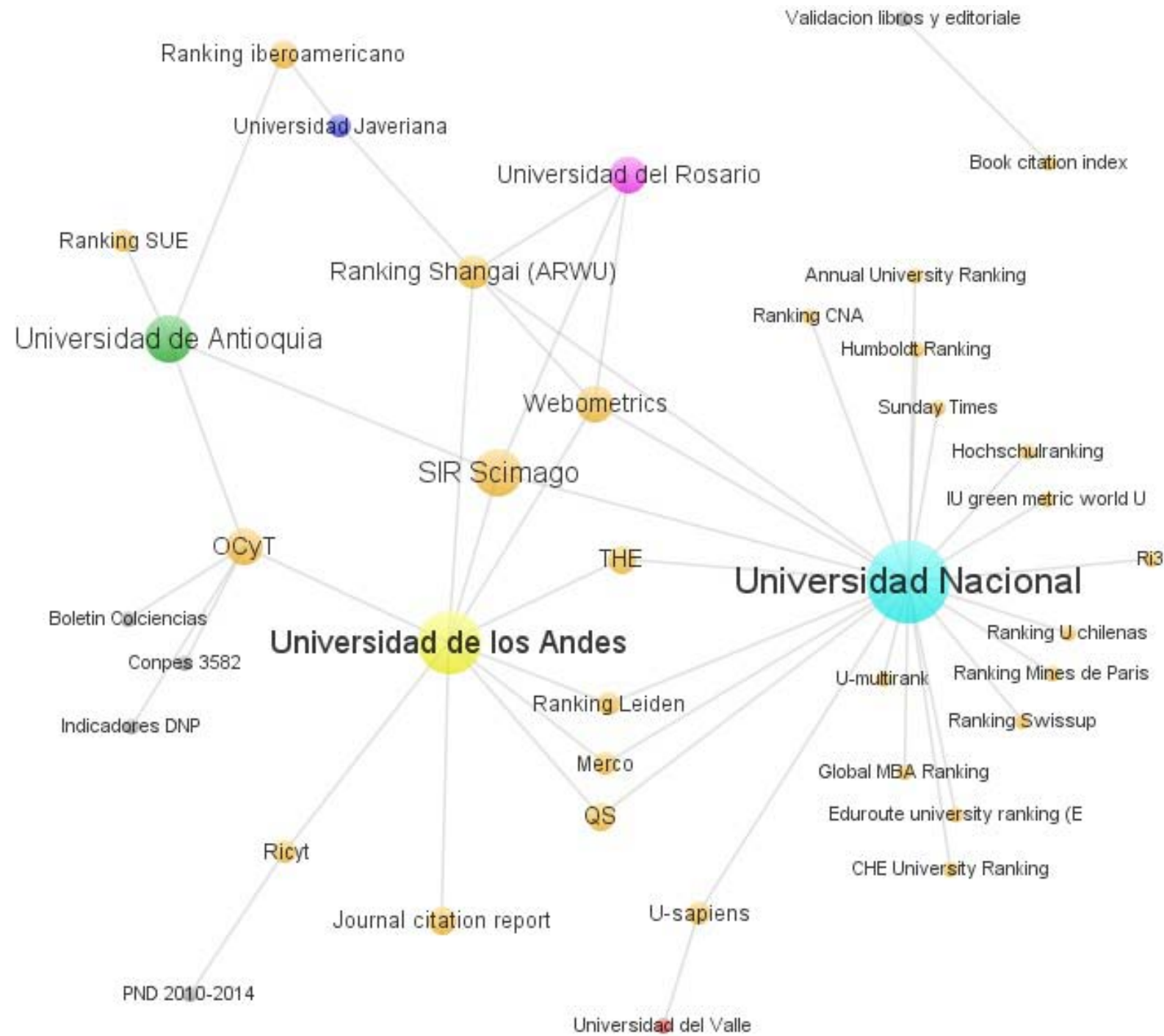

Elaboración propia.

Nodos de colores excepto naranja: Universidades. Nodos naranja: Rankings. Nodos grises: documentos nacionales. Relación: Citación de los ranking en documentos institucionales.

\section{DISCUSIÓN Y CONCLUSIONES}

Se pueden evidenciar tres fuerzas conflictivas: por un lado los sistemas de evaluación internacional jalonan la propuesta de considerar un núcleo muy pequeño de tipos de productos investigativos que pueden ser valorados como nuevo conocimiento (Web of Science y Scopus); por el otro lado están los sistemas de investigación de diferentes disciplinas no reconocidos que participan de los debates para la inclusión de sus productos; en medio los sistemas de reconocimiento de la producción científica como los de Colciencias y las Universidades. Estas tensiones plantean distintos escenarios para las políticas institucionales: A Colciencias la búsqueda de herramientas para evaluar la calidad de los productos incluidos dentro de su sistema ampliamente incluyente (4 formas diferentes de producir en investigación), en tanto el mercado internacional impulsa sólo a reconocer un grupo muy pequeño de tipos de producción (ni siquiera una variedad completa de formas productivas). Las disciplinas no experimentales no sólo tienen 
un problema de visibilidad para los instrumentos de medición, sino también una larga lucha por la inclusión de nuevos productos, pero también por su adaptación a nuevas formas productivas para sus campos. Así los sistemas de reconocimiento eventualmente tendrán que incluir las partituras (por ejemplo) como productos de investigación, pero esos grupos de investigación también deben publicar sus resultados en revistas evaluadas por pares. Las Universidades en ese sentido tienen que hacer un doble esfuerzo: generar sistemas bastante especializados de observación tanto de las formas de producción de la investigación más allá de lo reconocido por sistemas internacionales (Web of Science, Scopus, por ejemplo), y la exploración conceptual y metodológica de forma de medición del impacto de la investigación en la sociedad. Colciencias está generando mecanismos de vanguardia que jalonan a las Universidades en ese sentido al reconocer productos de apropiación social del conocimiento, formación investigativa, tecnología y relación con organizaciones sociales, así como la producción de nuevo conocimiento, todos entendidos por Colciencias como productos comunicativos de la investigación.

En esta discusión las áreas de artes, arquitectura y ciencias de la comunicación son especiales. Ellas podrían presentar mayor variedad en términos de tipos de productos o formatos diferentes de comunicación de resultados, generados a partir de sus procesos particulares de investigación, trabajo académico y creación. Sin embargo, aún está en proceso de validación en varios espacios del Sistema Nacional de Ciencia y Tecnología e Innovación; la Universidad del Valle hizo explícitos los tipos de productos que validan para esta área 4 años después del decreto 1279; y de la Universidad de los Andes identificamos un documento donde se veían avances de este proceso. Para el resto de instituciones sólo se conocen las tres categorías de productos artísticos que establece el decreto 1279, pero no cómo se está implementando en cada universidad.

El libro, que es una de las formas centrales de comunicación del conocimiento en humanidades y las ciencias sociales, encuentra una amplia aceptación y fuerte valoración en términos de lo que se declara en la documentación institucional pero el acceso y registro a esta información es muy incipiente, ya que las dos principales bases de datos internacionales (Web of Science y Scopus) empiezan a registrar los libros en un momento posterior al registro de revistas, y aún no tienen una cobertura suficientemente amplia. A nivel nacional el proceso de registro de editoriales tiene una experiencia importante con el sistema de Publindex de Colciencias. Este sistema es utilizado para asignación de puntajes y escalonamiento de los docentes, pero no para medir el impacto de la producción universitaria, lo cual se puede considerar una contradicción misma del sistema. España, por ejemplo, ya empieza a integrar este tipo de clasi- ficaciones de las editoriales en sus rankings (ver http://epuc.cchs.csic.es/SPI/).

Aunque Colciencias ha avanzado de manera importante en la definición conceptual y metodológica para el reconocimiento de diversas formas comunicativas de la investigación tanto los sistemas GrupLAC y CVLAC presentan problemas de validación de información. De este tipo de sistemas nacionales o regionales hay intentos diversos, inclusive con dificultades similares (Sanz Casado y otros, 2013), sin embargo su consolidación permitiría obtener datos más precisos y de carácter público. Mientras el sistema se acomoda, una salida alternativa puede ser apostar por la construcción de bases de datos institucionales que permitan hacer una medición más precisa de su producción. Estas bases de datos pueden estar en comunicación con las bases de datos de Colciencias en el futuro, pero eso dependerá únicamente de los acuerdos políticos a los que se logre llegar por parte de las instituciones.

Con respecto a los rankings, ya en la literatura científica se ha advertido sobre la importancia de diversificar las fuentes y contar con equipos especializados para leer rankings, bases de datos y otros mecanismos de vigilancia de la producción (Delgado, 2012; Torres y Cabezas, 2012). En este sentido no todas las Universidades mejor posicionadas en Colombia lo están haciendo. Suponemos que no es sólo un problema de las universidades colombianas, en tanto es tema de debate en las publicaciones sobre ciencias de la información. La dependencia de bases de datos de empresas comerciales pone en riesgo la toma de decisiones en cuanto al reconocimiento de diferentes formas de comunicación del conocimiento. De acuerdo con lo anterior, las acciones de la U. de los Andes o la U. Nacional permiten observar un conjunto de buenas prácticas que pueden explicar su visibilidad creciente: vigilancia en distintas bases, reflexividad sobre los datos en producción de documentos y consideración de productos de nuevo conocimiento en discusiones propuestas desde las dependencias administrativas de la investigación.

Los rankings internacionales están en su apogeo, pero desde el punto de vista productivo y de impacto de la investigación sus fuentes son limitadas. Sus indicadores sólo permiten observar el impacto de las Universidades más importantes en los campos experimentales. En un primer momento habría que aclarar que impacto no es igual a calidad (Pendlebury y Adams, 2012; Smith, 2012). Con esto aclarado habría que observar que la oferta de bases de datos está determinada por el mercado que es estrecho en tanto podría considerarse un duopolio. El producto ofrecido es vendido como la producción investigativa de la ciencia de mayor impacto. Sin embargo, encontramos en esta investigación gran cantidad de productos que no están incluidos en inventarios de producción internacional. $\mathrm{Si}$ a eso sumamos todas las voces de asociaciones 
nacionales, las críticas ya expuestas al inicio de este escrito y sobre todo la necesidad de entender el impacto real de la producción académica en Universidades por fuera de las 100 primeras, habría que hacer una revisión de las formas de lectura de todos estos rankings.

En esta investigación se evidencia que algunas Universidades deciden leer su desempeño a la luz de muchos rankings. Esto hace la diferencia entre trabajar para un ranking o que el ranking sea útil para el trabajo de los investigadores. Sin embargo no hay congruencia entre las bases de datos consultadas para verificar la funcionalidad de un grupo y su impacto. Mientras es posible encontrar gran variedad de productos y formas resultantes de procesos de investigación y creación de conocimiento, sólo se miden los artículos escritos, pero aquellos que aparecen sólo a través de selecciones y algoritmos realizados por los equipos de Elsevier, ISI Thompson o Google Scholar.

Finalmente, en términos de políticas institucionales y públicas, ¿por qué tomarse la molestia de revisar varios rankings, reconocer nuevos productos y pensar en la posibilidad de nuevos indicadores o formas de medición? Si la apuesta es tratar de entender qué están aportando al conocimiento y qué problemas están resolviendo los diferentes programas de investigación de Universidades y Centros de Investigación, la respuesta dada por un índice de impacto simplifica el potencial desarrollado. Esta investigación demuestra que en materia de políticas de evaluación, no todo el conocimiento desarrollado por Universidades colombianas está escrito en artículos ISI y Scopus, ni todo el impacto del conocimiento investigativo en la sociedad está determinado por los índices de impacto. El bache existente entre lo reconocido por las Universidades y los sistemas de medición con los que rigen su evaluación lanzan una alerta urgente sobre la necesaria reflexión acerca de lo que se entiende por el conocimiento aportado por las Universidades en una dimensión mucho más amplia que la ofrecida por los distintos índices de impacto, imperfectos en muchos aspectos y difíciles de delimitar en sus alcances.

\section{AGRADECIMIENTOS}

Este artículo se hace en el marco del proyecto "Línea base para la construcción de indicadores de comunicación del conocimiento producido por el área de ciencias sociales, humanidades y artes". Financiado por la Universidad de Antioquia. Convocatoria CODI Ciencias Sociales, Humanidades y Artes 2012. Participantes: Instituto de Estudios Regionales, Grupo de Investigación Redes y Actores Sociales, Grupo de Investigación de Psicología Social y Política. Se agradece el apoyo de las investigadoras Claudia Puerta Silva y Liliana Sánchez Mazo.

\section{BIBLIOGRAFÍA}

Archambault, E.; Larivière, V. (2010). Los límites de la bibliometría en el análisis de la literatura en ciencias sociales y humanidades. En UNESCO. Informe sobre las ciencias sociales en el mundo. Las brechas del conocimiento. ( $\mathrm{pp}$. 268-271). México.

Asociación Alemana de Sociología (2013). http:// www.soziologie.de/ Página Web [Consultada el 11 de Octubre de 2013]

Cabezas-Clavijo, Á. ; Torres-Salinas, D. (2012). Google scholar citations y la emergencia de nuevos actores en la evaluación de la investigación. Anuario ThinkEPI, 6, 147-153.

Colciencias. (2013a). Informe corto de resultados definición de grupos de investigación, tecnológica o de innovación año 2012. Recuperado 22 de febrero de 2013, a partir de http://www.colciencias.gov.co/scienti/

Colciencias. (2013b). Modelo de medición de Grupos de Investigación, Tecnológica y de Innovación. Recuperado a partir de http://www. colciencias.gov.co/sites/default/files/upload/ documents/documento_modelo_de_medicion_grupos_2013-version_ii_definitiva_ dic_10_2013._protected.pdf

Colectivo por la Ciencia (2013). Segunda carta por la ciencia. En: Sin Permiso: artículos en la web. Disponible en http://www.sinpermiso.info/ textos/index.php?id=6029 [Consultado el 3 de agosto de 2013].

Decreto Presidencial 1279. (2002, 19 de Junio). Diario Oficial, Imprenta Nacional de la República de Colombia 44.840. Junio 20, 2002.

De Nooy, W; Mrvar, A; Batagelj, V (2011). Exploratory Social Network Analysis with Pajek. Expanded Edition. Cambridge: Cambridge University Press. http://dx.doi.org/10.1017/ CBO9780511996368

Delgado López-Cózar, E. (2012). Cómo se cocinan los rankings universitarios. Dendra Médica. Revista de Humanidades, 11(1), 43-58.

Delgado-López-Cózar, E.; Cabezas-Clavijo, Á. (2013). Ranking journals: could Google Scholar Metrics be an alternative to Journal Citation Reports and Scimago Journal Rank? Learned Publishing, 26(2), 101-114. http://dx.doi. org/10.1087/20130206

Dorrë, K.; Lessenich, S.; Singo, I. (2013). Sociólogos alemanes proponen boicotear el ranking académico. Traducción de Sinpermiso. Original tomado de: http://www.isa-sociology.org/ global-dialogue/2013/04/german-sociologistsboycottacademic-ranking/ [Consultado el 5 de junio de 2013]

Estrada-Mejía, C.; Forero-Pineda, C. (2010). The quest for visibility of scientific journals in Latin America. Learned Publishing, 23(3), 237-252. http://dx.doi.org/10.1087/20100306

Freeman, L. (1978/1979). Centrality in Social Network Analysis: Conceptual Clarification. So- 
cial Networks, 1, pp. 215-239. http://dx.doi. org/10.1016/0378-8733(78)90021-7

Giménez Toledo, E.; Torres-Salinas,D. (2011). Book citation index: nueva historia sobre big science y little science. Anuario ThinkEPI, (1), 195-202.

Glänzel, W.; Schoepflin, U. (1999). A bibliometric study of references literature in the sciences and social sciences. Information Processing and Management 35, 31-44. http://dx.doi. org/10.1016/S0306-4573(98)00028-4

Hazelkorn, E (2010) Pros y contras de la evaluación de la investigación. En UNESCO. Informe sobre las ciencias sociales en el mundo. Las brechas del conocimiento. (pp. 268-271). México.

Macías Chapula, C.A. (2001). Papel de la infometría y de la cienciometría y su perspectiva nacional e internacional. ACIMED, 9.

Michels, C.; Schmoch, U. (2012). The growth of science and database coverage. Scientometrics, 93, 831-846. http://dx.doi.org/10.1007/ s11192-012-0732-7

Moreno Vargas, C. (2012). El arte de la investigación-creación. Pesquisa: Publicación de divulagación científica y tecnológica PUJ, 20.

Nederhof, A.J. (2006). Bibliometric monitoring of research performance in the Social Sciences and the Humanities: a review. Scientometrics, 66 (1), 81-100. http://dx.doi.org/10.1007/ s11192-006-0007-2

Pendlebury, D.A.; Adams, J. (2012). Comments on a critique of the Thomson Reuters journal impact factor. Scientometrics, (92), 395-401. http://dx.doi.org/10.1007/s11192-012-0689-6

Rodríguez Yunta, L. (2010). Las revistas iberoamericanas en Web of Science y Scopus: visibilidad internacional e indicadores de calidad. Comunicación presentada en el VII Seminario Hispano-Mexicano de Investigación en Bibliotecología y Documentación. Ciudad de México, 8 de abril de 2010.

Romero-Torres, M.; Acosta-Moreno, L.A.; TejadaGómez, M.A. (2013). Ranking de revistas científicas en Latinoamérica mediante el índice $\mathrm{h}$ : estudio de caso Colombia. Revista Española de Documentación Científica, 36(1). http://dx.doi. org/10.3989/redc.2013.1.876

Rubio Liniers, M.A. (1999). Bibliometría y ciencias sociales. Clío, 7.

Sánchez Torres, J.M.; Moreno Meneses, G.M. (2011). Caracterización de Ranking Universitarios. Recuperado a partir de https://99997f89-a62cb3a1a-s-sites.googlegroups.com/site/vicerrectoriavri/archivos/Caracterizaci\%C3\%B3n de Ranking Universitarios.pdf? [Consultado el 14 de Junio d 2013]

Sanz-Casado, E.; García-Zorita, C. ; Serrano-López, A.E.; Efraín-García, P.; De Filippo, D. (2013). Rankings nacionales elaborados a partir de múltiples indicadores frente a los de índices sintéticos. Revista Española de Documentación Científica, 36(3), e012. http://dx.doi.org/10.3989/ redc. 2013.3.1.023
Smith, D.R. (2012). Impact factors, scientometrics and the history of citation-based research. Scientometrics, 92(2), 419-427. http://dx.doi. org/10.1007/s11192-012-0685-x

Stichbe, R. (2003). Differentiation of Scientific disciplines: causes and consequences. Encyclopedia of Life Support Systems (EOLSS), UNESCO, Paris.

Torres-Salinas, D.; Cabezas-Clavijo, Á. (2012). Herramientas para la evaluación de la ciencia en universidades y centros $I+D$ : descripción y usos. Retrieved from http://digibug.ugr.es/handle/10481/20220 [Consultado el 1 de Septiembre de 2013]

Torres-Salinas, D.; Delgado-López-Cózar, E. (2013). Cobertura de las editoriales científicas del Book Citation Index en Ciencias Sociales y Humanidades: ¿La historia de repite? Anuario ThinkEPI, 7, 110-113.

Universidad de Antioquia. Área de Ciencias Sociales, Humanidades y Artes (2012). Productos de Nuevo Conocimiento. Documento de trabajo.

Vélez Cuartas, G.; Gómez Flórez, H.; Úsuga Ciro, A.; Vélez Trujillo, M. (2013a). Descripción de la dinámica estructural sobre la validación de productos de conocimiento en Colombia. IX Congreso Iberoamericano de Indicadores de Ciencia y Tecnología. Bogotá, Colombia: RICYT.

Vélez Cuartas, G.; Gómez Flórez, H. ; Úsuga Ciro, A.; Vélez Trujillo, M. (2013b). ¿Cómo valoran la producción académica las 6 universidades mejor escalafonadas del país? Revista Debates Vol. 65.

Villaveces Cardoso, J.L.; Orozco Castro, L.A.; Ruiz Ramos, C.F.; Chavarro Bohórquez, D.A.; Llanos Ballestas, E.J.; Silva Bohórquez, A.E.; Herrera Herrera, B. (2007). La Investigación en Uniandes 2006: Una Aproximación desde la Cienciometría. [Recuperado 19 de marzo de 2013], a partir de http://investigaciones.uniandes.edu. co/index.php/es/publicaciones

Villaveces Cardoso, J.L.; Orozco Castro, L.A.; Ruiz Ramos, C.F.; Chavarro Bohórquez, D.A.; Llanos Ballestas, E.J.; Silva Bohórquez, A.E.; Daza Caicedo, S.P. (2008). La investigación en Uniandes 2007: Elementos para una Política. [Recuperado 25 de marzo de 2013], a partir de http:// investigaciones.uniandes.edu.co/index.php/es/ publicaciones

Villaveces Cardoso, J.L.; Bello Ladino, D.H.; Delgado Vanegas, L.; Navas Calixto, A. C.; Zarur Latorre, F. (2012). La investigación en Uniandes: Perspectivas de la internacionalización. [Recuperado 12 de abril de 2013], a partir de http://investigaciones.uniandes.edu.co/index.php/es/publicaciones

Villaveces Cardoso, J.L.; Orozco Castro, L.A.; Chavarro Bohórquez, D.A.; Ruiz Ramos, C.F.; Bonilla Jiménez, R.; Bucheli Guerrero, V.A.; Delgado Vanegas, L. (2010). La investigación en Uniandes: construcción de una política. Bogotá, Colombia: Uniandes. [Recuperado 12 de abril de 2013] a partir de http://investigaciones.uniandes.edu. co/index.php/es/publicaciones 